\title{
OPEN Persistence and adherence to biologic therapies in juvenile idiopathic arthritis
}

\author{
Juan Carlos Nieto-González ${ }^{1 凶}$, Laura Trives-Folguera ${ }^{1}$, Alejandra Melgarejo-Ortuño², \\ Aranzazu Ais ${ }^{2}$, Belén Serrano-Benavente ${ }^{1}$, María Sanjurjo ${ }^{2}$, José María Álvaro-Gracia ${ }^{1} \&$ \\ Indalecio Monteagudo Sáez ${ }^{1}$
}

Juvenile idiopathic arthritis (JIA) is a chronic inflammatory disease that often requires biological therapy to control its activity. Medication persistence and adherence are important aspects on which we have scarce information. We performed a longitudinal, retrospective, and observational study based on data from the daily clinical management of JIA patients. We recorded clinical remission at 6 and 12 months. Persistence of biological therapy was evaluated using Kaplan-Meier curves, and adherence was assessed using the medication possession ratio (MPR). We included 68 patients who received biological therapy. Of these, $11(16.2 \%)$ and $5(7.4 \%)$ required a second and third drug, respectively. The persistence rate for biological therapy at 5 years was $64 \%$, with no differences between the first and second lines. Adherence was high during the first year of treatment (MPR80: 96.3\%) and also in the second and third years (MPR80: $85.2 \%$ and $91.8 \%$, respectively). Persistence and adherence to biological therapy were remarkably high in our JIA cohort. Adherence to biological treatments could be related to a higher probability of fulfilling the Wallace remission criteria at 6 months, although this was not confirmed at 12 months.

Juvenile idiopathic arthritis (JIA) is a heterogeneous disease that comprises all types of arthritis lasting longer than 6 weeks in patients under the age of 16 years $^{1}$. Treatment should be adjusted to each JIA subcategory, although many patients need systemic drugs ${ }^{2}$. These include synthetic disease-modifying antirheumatic drugs (sDMARDs), mainly methotrexate ${ }^{3}$, and biological therapy (bDMARDs), mostly tumor necrosis factor alpha (TNFa) inhibitors ${ }^{4,5}$.

Polyarticular JIA requires bDMARDs in around $50 \%$ of cases to reach the treatment goal ${ }^{6}$, which is remission ${ }^{7,8}$. However, more important than choosing a specific treatment per se is the requirement to follow a treat-to-target (T2T) approach, which improves disease activity irrespective of the systemic drugs used ${ }^{8-10}$.

Adherence is the extent to which a patient acts in accordance with medical prescriptions or recommendations ${ }^{11}$. According to the World Health Organization (WHO), adherence stands at around 50\% for chronic disease, both in adults and children, and plays a key role in the efficacy of treatment ${ }^{11}$. In children, adherence is difficult to measure owing to factors such as parental responsibility. However, in patients taking bDMARDs, adherence can be calculated using the medication possession ratio (MPR), which is based on the electronic dispensing record kept by the hospital pharmacy service.

Adherence has been sometimes evaluated by calculating medication persistence ${ }^{12}$, although these concepts differ. Persistence is the entire time that the patient continues to take the treatment and ends when the patient stops the treatment for any reason ${ }^{13-17}$. Compared with adult data on bDMARD persistence, many JIA patients stop their medication owing to clinical remission, thus potentially explaining differences in persistence ${ }^{12-17}$.

The aim of our study was to calculate adherence to bDMARDs in our JIA cohort during the first 3 years of treatment. We also aimed to calculate drug persistence for bDMARDs.

\section{Methods}

We conducted a longitudinal, observational, and retrospective study of our JIA cohort. The study was carried out in accordance with relevant guidelines and regulations, and its design was in line with current regulations and with the approval of the Spanish Agency of Medicines and Medicinal Products. We included all JIA subcategories

${ }^{1}$ Rheumatology Department, Hospital General Universitario Gregorio Marañón, Madrid, Spain. ${ }^{2}$ Pharmacy Department, Hospital General Universitario Gregorio Marañón, Madrid, Spain. ${ }^{\square}$ email: juancarlos.nietog@ gmail.com 


\begin{tabular}{|c|c|c|c|c|}
\hline & $\begin{array}{l}\text { All patients } \\
\mathrm{n}: 68\end{array}$ & $\begin{array}{l}\text { Remission at } 12 \text { months } \\
\mathrm{n}: 58\end{array}$ & $\begin{array}{l}\text { Not remission at } 12 \text { months } \\
\mathrm{n}: 10\end{array}$ & $p$ \\
\hline Female sex, n (\%) & $39(57.4 \%)$ & $36(94.7 \%)$ & $2(5.3 \%)$ & 0.013 \\
\hline Age, mean (SD) & $6.6(4.7)$ & $6.6(4.5)$ & $8.9(4.7)$ & 0.192 \\
\hline \multicolumn{5}{|l|}{ JIA subcategory } \\
\hline Systemic & $5(7.4 \%)$ & $5(100 \%)$ & 0 & \multirow{5}{*}{0.508} \\
\hline Oligoarticular & $29(42.6 \%)$ & $22(84.6 \%)$ & $4(15.4 \%)$ & \\
\hline Polyarticular & $19(27.9 \%)$ & $16(88.9 \%)$ & $2(11.1 \%)$ & \\
\hline Enthesitis-related & $11(16.2 \%)$ & $15(88.2 \%)$ & $2(11.8 \%)$ & \\
\hline Psoriatic & $4(5.9 \%)$ & & & \\
\hline ANA-positive & $27(39.7 \%)$ & $21(87.5 \%)$ & $3(12.5 \%)$ & 0.704 \\
\hline Chronic anterior uveitis & $7(10.3 \%)$ & $6(100 \%)$ & 0 & 0.224 \\
\hline 1st biologic & $68(100 \%)$ & $41(85.4 \%)$ & $7(14.6 \%)$ & \multirow{3}{*}{0.970} \\
\hline 2nd biologic & $11(16.2 \%)$ & $9(81.8 \%)$ & $2(18.2 \%)$ & \\
\hline 3rd biologic & $5(7.4 \%)$ & $4(80 \%)$ & $1(20 \%)$ & \\
\hline
\end{tabular}

Table 1. Demographic data of patients with juvenile idiopathic arthritis and comparison between groups by remission at 12 months with their first biologic therapy. JIA, juvenile idiopathic arthritis; MPR, medication possession ratio; ANA, antinuclear antibodies; SD, standard deviation.

and all bDMARDs. We excluded patients who were treated with only steroids and/or sDMARDs and patients who were naïve for bDMARDs. We also excluded patients with less than 6 months of treatment from the adherence calculation. All patients and parents agreed to participate in the study, and informed consent was provided by the parents or patients when applicable. The study was approved by the local ethics committee (Comité de Ética de la Investigación con Medicamentos, Hospital General Universitario Gregorio Marañón, Madrid, Spain).

We collected clinical and laboratory data from the electronic medical records. We reviewed the start and end dates for biological therapy and the reasons for ending therapy. The main reasons for ending a bDMARD were reaching remission, appearance of adverse events, patient or parental request, and financial aspects. We also reviewed clinical remission at 6 and 12 months after initiation of a biological treatment.

The MPR was calculated based on data from our pharmacy registry of biologic therapy obtained from electronic prescriptions as the number of doses dispensed in relation to the dispensing period. It is usually expressed as a percentage. We calculated the annual adherence to the first line of biologics during the first 3 years of treatment.

For practical and statistical reasons and based on pathophysiology, we created 4 homogeneous groups of JIA; group 1, oligoarticular JIA (including persistent and extended subcategories); group 2, polyarticular JIA (including rheumatoid factor-positive and -negative subcategories); group 3, juvenile spondyloarthritis, (including enthesitis-related arthritis and psoriatic arthritis); and group 4, systemic JIA. We used these groups to compare persistence and clinical data between the different subcategories.

\section{Definitions}

$>$ Lack of adherence: Lack of adherence to bDMARDs was defined as an MPR lower than $80 \%$ per year $(\mathrm{MPR}<80)^{18}$. We also applied a more exacting criterion by defining lack of adherence as an MPR lower than $90 \%$ per year $(\mathrm{MPR}<90)$.

An adherence of $80 \%$ or higher is represented as MPR80 and MPR90 for adherence of $90 \%$ of higher.

$>$ Clinical remission: Patient fulfilling the Wallace criteria with or without treatment. No arthritis, enthesitis, dactylitis, uveitis, or systemic features, such as fever.

\section{Statistics}

Data for continuous variables are expressed as mean and standard deviation (SD) or median and interquartile range (IQR). Data for qualitative variables are expressed as frequencies and percentages. Biological persistence was studied using Kaplan-Meier curves. Comparisons between groups were made using the Pearson chi-square test, $t$ test, or Mann Whitney test, as applicable. All statistical tests were performed 2-sided, and a p-value of $<0.05$ was considered statistically significant. All calculations were performed using IBM SPSS Statistics for Windows, Version 25.0 (Armonk, NY: IBM Corp).

\section{Results}

From a total of 132 JIA patients followed in our unit, we included 68 patients $(51.5 \%)$ who had received 1 or more bDMARDs. Of these, $11(16.2 \%)$ needed a second bDMARD and 5 (7.4\%) a third bDMARD. Therefore, in total we analyzed 84 exposures to biologics: 72 (85.7\%) with TNFa inhibitors, 8 (9.5\%) with interleukin-6 inhibitors, and 4 (4.8\%) with other bDMARDs. Table 1 summarizes the demographic data for the study population according to whether or not they had reached remission at 12 months.

The most common bDMARD was etanercept, which was taken by 52 patients (76.5\%), followed by adalimumab in 9 patients $(13.2 \%)$, tocilizumab in 3 patients $(4.4 \%)$, anakinra in 2 patients $(2.9 \%)$, and infliximab and 
a

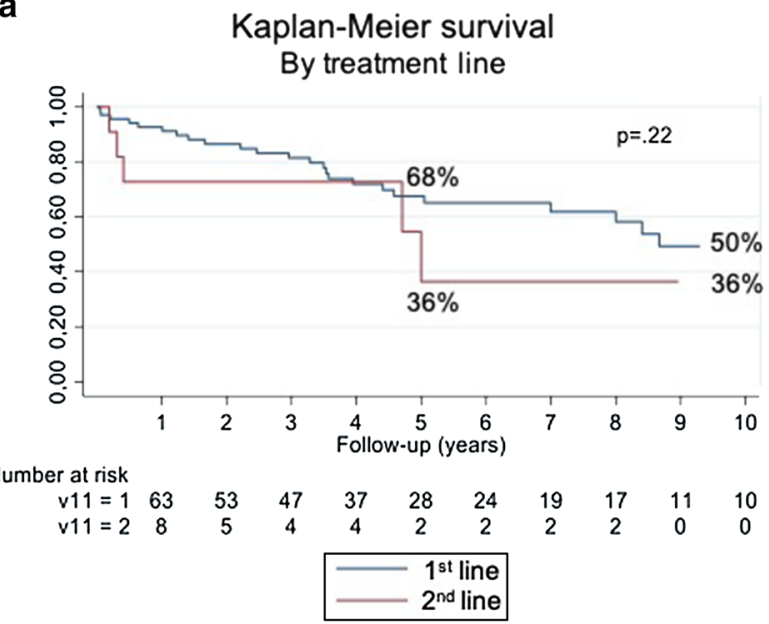

b
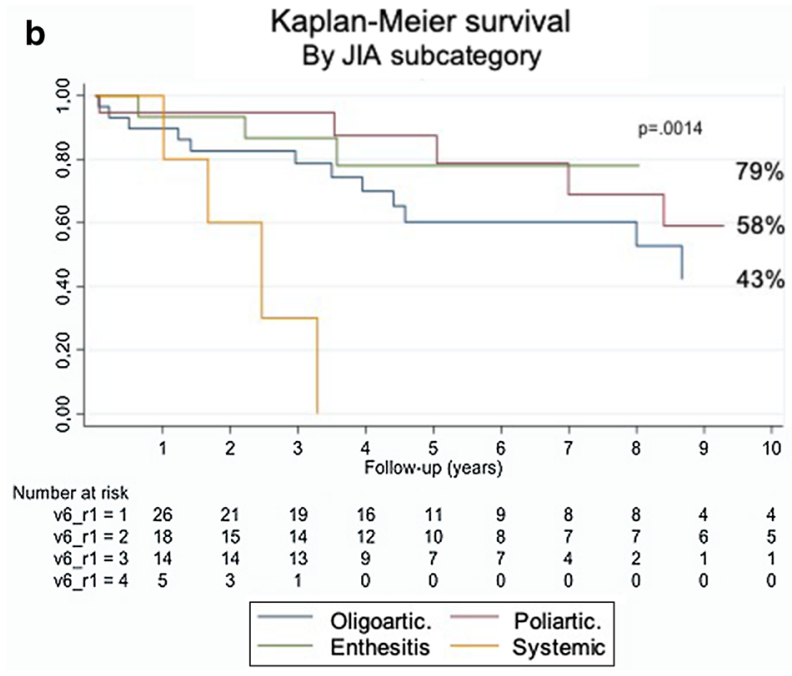

Figure 1. Kaplan-Meier curves for persistence of biologics by line of treatment (a) and by JIA subcategory (b).

ustekinumab in 1 patient (1.5\%) each. The mean time to starting a bDMARD was 1.1 years (interquartile range [IQR] 0.3-3.2), and the mean time to tapering of the first biologic was 0.9 years (IQR 0.5-1.2). We found that female patients had a higher probability of achieving remission at 12 months than male patients (p:0.013), with no other differences for the JIA subcategory (p:0.617), uveitis (p:0.224), or antinuclear antibody (ANA) status (p:0.704).

Persistence of biologic therapy. We calculated the overall persistence of biologic therapy at 5 years and compared the persistence of first- and second-line agents over a 10-year follow-up period. The overall persistence after 5 years of follow-up was $64 \%$. There were no differences in persistence between the first and second lines of biologic treatment. However, persistence of biologics in the systemic JIA (group 4) was significantly shorter, with no differences between the rest of the JIA subcategories. The Kaplan-Meier curves for the first- and second-line biologics and for the different JIA subcategories are shown in Fig. 1.

The reasons for discontinuation of biologics at any time (31 patients, 36.9\%), were remission in 15 cases (48.4\%), inefficacy in 9 cases $(29 \%)$, adverse events in 4 cases $(12.9 \%)$, and other causes in 3 cases $(9.7 \%)$. The mean time of bDMARD treatment before stopping medication ( 15 cases) due to remission was 4.1 years, and all but 2 cases continued with methotrexate as a systemic treatment after stopping bDMARDs.

Adherence to biologic therapy. Adherence to bDMARDs at 1 year was $96.3 \%$ with MPR80 and $86.3 \%$ with MPR90. The percentages of adherence in patients who continued the treatment were similar after 2 and 3 years, although they decreased slightly over time. Figure 2 shows adherence over 3 years of therapy with a biologic. There were no differences between adherent and non-adherent patients, irrespective of whether they were divided by JIA subcategory (p:0.972), sex (p:0.398), or ANA status (p:0.217).

We evaluated whether adherence to the biologic had any influence on the possibility of achieving clinical remission at 6 and 12 months (Table 2). When we selected MPR80, statistically significant differences were observed for remission at 6 months (p:0.039) but not at 12 months (p:0.153). Moreover, these differences were not observed when we selected MPR90 (p:0.144 and 0.274 respectively).

\section{Discussion}

We usually follow a T2T strategy in the management of JIA, exactly as we do in adult patients with chronic arthritis. Our study showed high remission rates, persistence, and adherence to biological therapy in JIA. This T2T strategy, highly recommended by international task forces ${ }^{10}$, might be the reason for such favorable results. We found a remission to be more frequent in female than in male patients; however, this is not easy to explain, because sex has not been reported to affect disease severity, treatment response, or adherence.

Around $50 \%$ of the patients needed biologic therapy to control disease activity, and $16 \%$ and $7.6 \%$ needed a second and third biologic, respectively. Our results are similar to those of international studies in which the authors also followed a T2T strategy ${ }^{6,8,9}$. However, the clinical response in our cohort was slightly better, with complete remission after 6 months in more than three quarters of the patients, increasing to more than $80 \%$ at 12 months. A recent study showed $48 \%$ of patients to be in remission at month 12 after following a T2T strategy ${ }^{8}$, although the authors reported that $37 \%$ of patients were taking bDMARDs compared with $51.5 \%$ in our study, thus explaining in part the differences in findings. Moreover, a systematic literature review estimated that up to $68 \%$ of patients reached remission after 12 months of treatment ${ }^{19}$.

Persistence of biological therapy. Follow-up was long for most of the patients, and we were able to calculate the persistence of biologics until 10 years of follow-up in some cases. Persistence at 5 years was remarkably high, with numerical but not statistical differences between the first and second lines of treatment. One study 


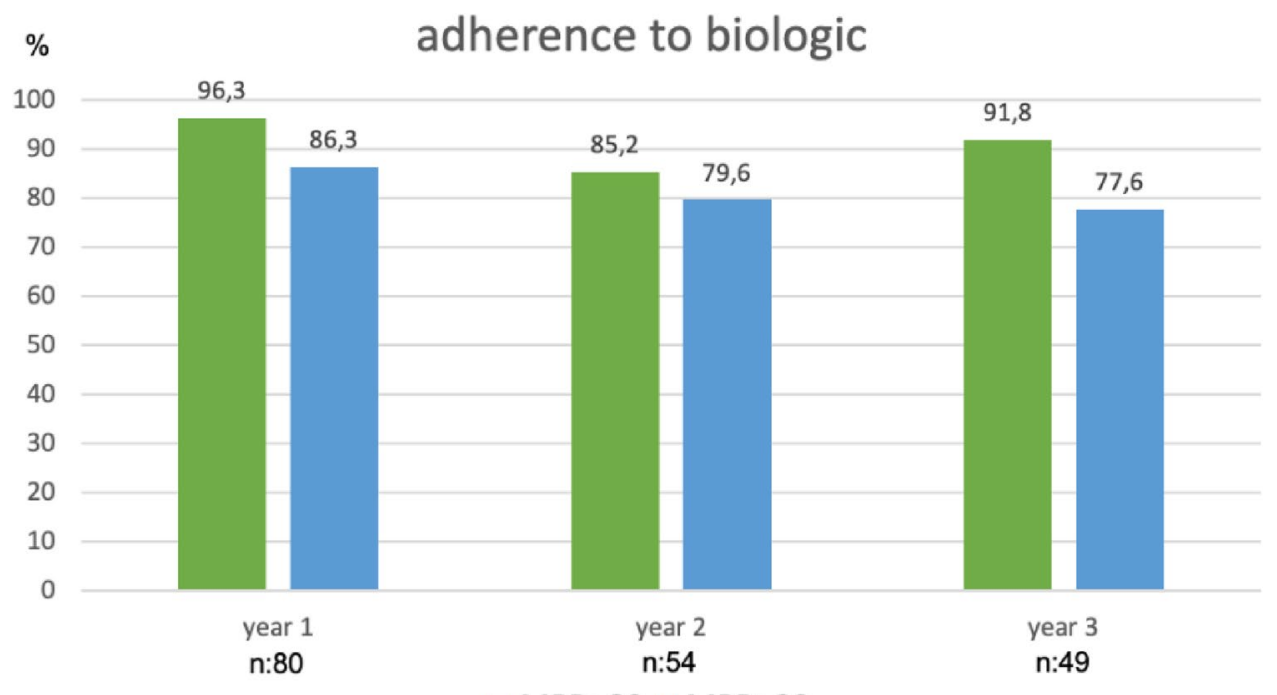

MPR $>80 \square \mathrm{MPR}>90$

Figure 2. Adherence to therapy with a biologic during the first 3 years of treatment with a medication possession ratio (MPR) greater than $80 \%$ and $90 \%$.

\begin{tabular}{|c|c|c|c|c|c|c|c|}
\hline & \multicolumn{3}{|c|}{ Remission at 6 months (n:80) } & & \multicolumn{3}{|c|}{ Remission at 12 months (n:68) } \\
\hline & Yes (n:64) & No (n:16) & $\mathrm{p}$ & & Yes (n:58) & No $(n: 10)$ & $\mathbf{p}$ \\
\hline \multicolumn{8}{|l|}{ 1st year (n:80) } \\
\hline MPR80 (n:77) & $63(81.8 \%)$ & $14(18 \%)$ & \multirow{2}{*}{0.039} & MPR80 (n:66) & $57(86.4 \%)$ & $9(13.6 \%)$ & \multirow{2}{*}{0.153} \\
\hline MPR < $80(n: 3)$ & $1(33 \%)$ & $2(66.7 \%)$ & & MPR < 80 (n:2) & $1(50 \%)$ & $1(50 \%)$ & \\
\hline MPR90 (n:69) & $57(82.6 \%)$ & $12(17.4 \%)$ & \multirow{2}{*}{0.144} & MPR90 (n:61) & $53(86.9 \%)$ & $8(13.1 \%)$ & \multirow{2}{*}{0.274} \\
\hline MPR $<90(\mathrm{n}: 11)$ & $7(63.6 \%)$ & $4(36.4 \%)$ & & MPR < 90 (n:7) & $5(71.4 \%)$ & $2(28.6 \%)$ & \\
\hline
\end{tabular}

Table 2. Remission (Wallace criteria) at 6 and 12 months after initiation of biologic therapy with respect to adherence to treatment during the first year. In bold are showed the group differences statistically significant.

reported persistence of around $50 \%$ at 1 year ${ }^{18}$, and another study reported a higher persistence of $50-60 \%$ for TNFa inhibitors at 5 years ${ }^{12}$. Our results were higher and similar to those of a German cohort ${ }^{12}(89.9 \%$ at 1 year and $64 \%$ at 5 years). It is also very interesting that approximately $50 \%$ of patients discontinued biologic treatment due to remission, although elsewhere, only around 9-13\% of JIA patients discontinued TNFa inhibitors due to remission ${ }^{12,14,16}$

Adherence to biological therapy. Adherence to therapy is essential if the clinical response is to be good $^{11}$. The WHO estimated adherence to chronic treatments to be approximately $50 \%{ }^{11}$ in adults and children. Previous studies reported maximum adherence (MPR80) to TNFa inhibitors of $42.1 \%{ }^{17}$, and a recent literature review of adherence to treatment in JIA showed a maximum adherence of $65 \%{ }^{19}$. However, results were higher (MPR80 96.3\%) in our JIA cohort, which could be partially explained by the important efforts made by our Pharmacy Department during follow-up to optimize therapy with bDMARDs. Adherence can be limited by numerous barriers (eg, the patient, the patient's family, and the kind of drugs ${ }^{20}$ ), which must be taken into consideration if we are to improve it.

Since we had remarkably high adherence results, we applied more exacting criteria by using MPR90. The proportion of adherent patients was still higher than in previous reports at 1 year (MPR90: 86.3\% vs. MPR80: $38.4 \%)^{17}$. Moreover, the high adherence to biologic treatment was also maintained in our cohort during the second and third years.

Based on the Wallace remission criteria at 6 and 12 months, MPR $<80$ was related to a reduced probability of achieving a remission at 6 months, although this was not confirmed at 12 months, probably owing to the small number of patients with active disease at this time. At 6 months 3 out of 77 patients $(3.89 \%)$ had a MPR $<80$ and the proportion was even smaller at 12 months, 2 out of 66 patients (3\%).

Limitations of our study. The major limitation of our study is the small sample size in a very heterogeneous disease such as JIA. It would be interesting to analyze each subcategory separately and compare them with each other. To do so, it is imperative that collaborative studies be designed to ensure sufficient patients in each 
JIA subcategory. Our study is also limited by its observational and retrospective study design, although this is the usual approach when studying persistence of and adherence to treatment.

\section{Conclusions}

Persistence of and adherence to biologic treatment in JIA were very good in our cohort. While the reasons for discontinuing biologic treatment vary, we found remission to be a frequent motive. Adherence to biologic treatments could be related to a higher probability of fulfilling the Wallace remission criteria at 6 months; however, this was not confirmed at 12 months.

Received: 25 March 2021; Accepted: 14 July 2021

Published online: 10 August 2021

\section{References}

1. Petty, R. E. et al. International League of Associations for Rheumatology classification of juvenile idiopathic arthritis: Second Revision, Edmonton 2001. J. Rheumatol. 31, 390-392 (2004).

2. Beukelman, T. et al. 2011 American College of Rheumatology recommendations for the treatment of juvenile idiopathic arthritis: initiation and safety monitoring of therapeutic agents for the treatment of arthritis and systemic features. Arthritis Care Res. (Hoboken) 63, 465-482 (2011).

3. Giannini, E. H. et al. Methotrexate in resistant juvenile rheumatoid arthritis. Results of the U.S.A.-U.S.S.R. double-blind, placebocontrolled trial. The Pediatric Rheumatology Collaborative Study Group and The Cooperative Children's Study Group. N. Engl. J. Med. 326, 1043-1049 (1992).

4. Lovell, D. J. et al. Etanercept in children with polyarticular juvenile rheumatoid arthritis. Pediatric Rheumatology Collaborative Study Group. N. Engl. J. Med. 342, 763-769 (2000).

5. Lovell, D.J., Ruperto, N., Goodman, S., Reiff, A., Jung, L., Jarosova, K. et al.; Pediatric Rheumatology Collaborative Study Group; Pediatric Rheumatology International Trials Organisation. Adalimumab with or without methotrexate in juvenile rheumatoid arthritis. N. Engl. J. Med. 359, 810-820 (2008).

6. Kearsley-Fleet, L., Heaf, E., Davies, R., Baildam, E., Beresford, M.W., Foster, H.E. et al.; BCRD and BSPAR-ETN study groups. Frequency of biologic switching and the outcomes of switching in children and young people with juvenile idiopathic arthritis: a national cohort study. Lancet Rheumatol. 2, e217-e226 (2020).

7. Wallace CA, Ruperto N, Giannini E; Childhood Arthritis and Rheumatology Research Alliance; Pediatric Rheumatology International Trials Organization; Pediatric Rheumatology Collaborative Study Group. Preliminary criteria for clinical remission for select categories of juvenile idiopathic arthritis. J. Rheumatol. 31, 2290-2294 (2004).

8. Wallace, C. A. et al. Trial of early aggressive therapy in polyarticular juvenile idiopathic arthritis. Arthritis Rheum. 64, 2012-2021 (2012).

9. Klein, A., Minden, K., Hospach, A., Foeldvari, I., Weller-Heinemann, F., Trauzeddel, R. et al. Treat-to-target study for improved outcome in polyarticular juvenile idiopathic arthritis. Ann Rheum Dis. 79, 969-974 (2020).

10. Ravelli, A. et al. Treating juvenile idiopathic arthritis to target: recommendations of an international task force. Ann Rheum Dis. $77,819-828$ (2018).

11. Adherence to Long-term Therapies: Evidence for Action, WHO 2003. IBSN 9241545992.

12. Horneff, G. et al. Comparison of treatment response, remission rate and drug adherence in polyarticular juvenile idiopathic arthritis patients treated with etanercept, adalimumab or tocilizumab. Arthritis Res. Ther. 18, 272 (2016).

13. Bethencourt Baute, J. J. et al. Persistence and adverse events of biological treatment in adult patients with juvenile idiopathic arthritis: results from BIOBASADER. Arthritis Res. Ther. 20, 227 (2018).

14. Romano, M. et al. Drug survival and reasons for discontinuation of the first course of biological therapy in 301 juvenile idiopathic arthritis patients. Reumatismo 65, 278-295 (2013).

15. Kearley-Fleet, L., Vicente González, L., Steinke, D. et al. Methotrexate persistence and adverse drug reactions in patients with juvenile idiopathic arthritis. Rheumatology (2019).

16. Mourao, A. et al. Effectiveness and long-term retention of anti-tumour necrosis factor treatment in juvenile and adult patients with juvenile idiopathic arthritis: data from Reuma.pt. Rheumatology 55, 697-703 (2016).

17. Favalli, E. G. et al. Real-life 10-year retention rate of first-line anti-TNF drugs for inflammatory arthritis in adult- and juvenileonset populations: similarities and differences. Clin. Rheumatol. 36, 1747-1755 (2017).

18. Ringold, S., Grant, S., Girdish, C., Vallace, C. \& Sullivan, S. Methotrexate and injectable tumor necrosis factor-alpha inhibitor adherence and persistence in children with rheumatic diseases. J. Rheumatol. 40, 80-86 (2013).

19. Shoop-Worrall, S. J. W., Kearsley-Fleet, L., Thomson, W., Verstappen, S. M. M. \& Hyrich, K. L. How common is remission in juvenile idiopathic arthritis: a systematic review. Semin Arthritis Rheum. 47, 331-337 (2017).

20. Favier, L. A. et al. Barriers to adherence in juvenile idiopathic arthritis: A multicenter collaborative experience and preliminary results. J Rheumatol. 45, 690-696 (2018).

\section{Author contributions}

J.C.N. and I.M. designed the study and analized the results. L.T.-F., A.M.-O. and B.S.-B. collected clinical data and all the authors reviewed and approved the results and the latest manuscript.

\section{Competing interests}

The authors declare no competing interests.

\section{Additional information}

Correspondence and requests for materials should be addressed to J.C.N.-G.

Reprints and permissions information is available at www.nature.com/reprints.

Publisher's note Springer Nature remains neutral with regard to jurisdictional claims in published maps and institutional affiliations. 
(c) (i) Open Access This article is licensed under a Creative Commons Attribution 4.0 International cc) License, which permits use, sharing, adaptation, distribution and reproduction in any medium or format, as long as you give appropriate credit to the original author(s) and the source, provide a link to the Creative Commons licence, and indicate if changes were made. The images or other third party material in this article are included in the article's Creative Commons licence, unless indicated otherwise in a credit line to the material. If material is not included in the article's Creative Commons licence and your intended use is not permitted by statutory regulation or exceeds the permitted use, you will need to obtain permission directly from the copyright holder. To view a copy of this licence, visit http://creativecommons.org/licenses/by/4.0/.

(C) The Author(s) 2021 